\title{
Lattice design and beam dynamics of a storage ring for a Thomson scattering $x$-ray source
}

\author{
Tenghui Rui and Wenhui Huang* \\ Accelerator Laboratory, Department of Engineering Physics, Tsinghua University, Beijing 100084, China
}

(Received 11 June 2018; published 19 October 2018)

\begin{abstract}
TTX-II (Tsinghua Thomson Scattering X-Ray Source Phase II) is a Thomson scattering based x-ray source currently under development at the accelerator laboratory in Tsinghua University. The circumference of the storage ring is $5.668 \mathrm{~m}$. The compact nature of the ring gives rise to several design challenges such as chromaticity correction and collective instability, which can be suppressed by carefully optimizing ring parameters. The lattice design and the beam dynamics of TTX-II are presented in this work.
\end{abstract}

DOI: 10.1103/PhysRevAccelBeams.21.100101

\section{INTRODUCTION}

Thomson scattering between laser beam photons and moderately relativistic electrons provides a way to generate $\mathrm{x}$-ray photons in a relatively simple setup. Though the photon flux and beam brightness of the Thomson scattering scheme may not be competitive against those of synchrotron sources, it has several attractive characteristics. The high energy versus angle correlation makes it easy to obtain a quasimonochromatic beam with a small bandwidth. For example, the bandwidth of the Tsinghua Thomson Scattering X-Ray Source (TTX) is 10\% [1]. The photon energy can be tuned by several ways: by varying electron beam energy, laser frequency, or the interaction angle. The circumference of ring-based Thomson scattering light sources can be as short as a few meters, which makes it an excellent choice for small facilities like hospitals.

Previously, there was a design of a storage ring for a Thomson scattering light source with a circumference of $4.8 \mathrm{~m}$ [2]. The ring consists of 4 dipole magnets with small bending radius of $0.25 \mathrm{~m}$ and large edge angles of $37^{\circ}$ to provide vertical focusing. In doing so, the nonlinearity induced by large edge angles contributes a significant amount to vertical chromaticity. In this previous study, the calculation of chromaticity only took into account the effect of quadrupole error and the hard edge fringe field of dipoles, which is not applicable when the bending angle and edge angles are large. The vertical chromaticity is estimated to be -10 after adding the contribution of the

\footnotetext{
*huangwh@mail.tsinghua.edu.cn

Published by the American Physical Society under the terms of the Creative Commons Attribution 4.0 International license. Further distribution of this work must maintain attribution to the author(s) and the published article's title, journal citation, and DOI.
}

edge angle. Compared with the previous results, the vertical chromaticity is increased by a factor of 10 . With a large and uncorrected chromaticity, momentum acceptance is as small as $0.4 \%$ and the Touschek lifetime is less than 180 sec. Also, head-tail instability arises quickly, and the actual beam lifetime would be much shorter than Touschek lifetime. The growth time of head-tail instability is no more than a few tens of microseconds. With limited space due to the compact design, active damping of headtail instability with feedback kickers is not possible. Some technical problems arise during later development as well. For example, the straight section supposed to accommodate the rf cavity is not long enough. The injection scheme is over optimistic about the injection angle. The beam injection trajectory crosses two bending magnets instead of the one that is intended, which results in difficulty of injection.

As a result of the beam dynamics problems and technical problems, we designed a new ring based on the old one, whose key components like rf cavity, magnets, and kickers are under construction at the accelerator laboratory in Tsinghua University. The design of TTX Phase II (TTX-II) is presented in this work. The remainder of this paper is organized as follows: Section II discusses the TTX-II lattice. Section III is focused on beam dynamics. The discussions and results are summarized in Sec. IV.

\section{LATTICE}

\section{A. Main requirements of the lattice}

The main goal of this storage ring is to store electron beams for Thomson scattering. Currently, the number of $\mathrm{X}$-ray photons generated by TTX is about $1 \times 10^{7}$ photons per shot at a repetition rate of $10 \mathrm{~Hz}$. We aim to increase the photon yield with TTX-II by a factor of 100, to about $1 \times 10^{10}$ photons per second. Therefore, the beam size at 
the interaction point should be optimized to be as small as possible, and the beam lifetime should be long enough to provide a stable photon yield for experiments or application.

The general consideration of the ring layout is to make the ring circumference as short as possible, with enough space for all equipment and some flexibility as far as the working point is concerned. The circumference of the ring is chosen to be $5.668 \mathrm{~m}$ to match beam revolution frequency with synchronizing system frequency $(2856 \mathrm{MHz})$ and to provide straight sections long enough for other equipment.

The dispersion at the sections where we place the interaction point and rf cavity not only contributes to the beam size but also causes a shift in closed orbit when particles pass through the rf cavity or interact with the laser and the longitudinal momentum changes. Hence, the dispersion function at these locations should be zero to ensure beam stability.

Natural chromaticity if not corrected with sextupoles will limit dynamic aperture, and thus beam lifetime. Moreover, without any damping mechanism, the lowest mode of head-tail instability destabilizes the beam quickly. It is difficult to correct natural chromaticity with a conventional scheme in TTX-II as will be discussed in the following section, so the natural chromaticity should be small enough not to cause beam instability.

The requirements of the TTX-II lattice are summarized as follow: The dispersion at IP and the rf cavity should be zero. Natural chromaticity should be as small as possible to ensure stability and beam lifetime. Betatron function at IP should be small to reduce beam size and enhance photon yield.

\section{B. Optics design}

To suppress the dispersion function at places where IP and the rf cavity is located, and the double bend achromat (DBA) structure is adopted. The ring consists of four bending magnets defining the beam trajectory. Each pair of the bending magnets along with three quadrupoles between them forms a DBA. The remaining two straight sections are dispersion free, and accommodate a stripline kicker for beam injection or extraction, and a rf cavity for longitudinal focusing. The kicker also serves as the interaction chamber for the electron beam and laser. The 3D schematics of the lattice is shown in Fig. 1.

Because of the small radius of the dipole magnets, the edge-field effects play an important role in transverse focusing and chromatic effects. The arc length and edge angles of the dipoles should be optimized to provide proper transverse focusing while keeping chromatic effects as small as possible.

For a bending magnet with a bending curvature of $h$ and an edge angle of $\theta$ on both ends, the contribution of the edge focusing to the chromaticity can be described by the following formulas: [3]

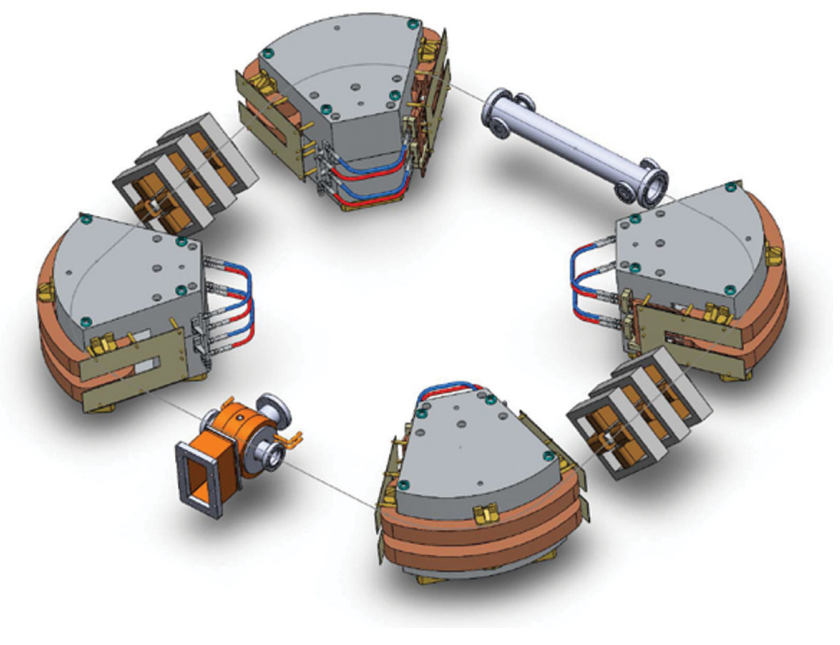

FIG. 1. Layout of TTX-II.

$$
\begin{aligned}
\Delta \xi_{x, 1}= & \frac{-h \beta_{x} \tan \theta}{-4 \pi}+\frac{h \tan ^{2} \theta\left(\beta_{x} \eta_{x}^{\prime}-\alpha_{x} \eta_{x}+h \eta_{x} \beta_{x} \tan \theta\right)}{-4 \pi} \\
\Delta \xi_{y, 1}= & \frac{h \beta_{x} \tan \theta}{-4 \pi} \\
& +\frac{-h \tan ^{2} \theta\left(\beta_{y} \eta_{x}^{\prime}-\alpha_{y} \eta_{x}-h \eta_{x} \beta_{y} \tan \theta\right)-\beta_{y} h \eta_{x}^{\prime}}{-4 \pi}, \\
\Delta \xi_{x, 2}= & \frac{-h \beta_{x} \tan \theta}{-4 \pi}+\frac{-h \tan ^{2} \theta\left(\beta_{x} \eta_{x}^{\prime}-\alpha_{x} \eta_{x}-h \eta_{x} \beta_{x} \tan \theta\right)}{-4 \pi}, \\
\Delta \xi_{y, 2}= & \frac{h \beta_{x} \tan \theta}{-4 \pi} \\
& +\frac{h \tan ^{2} \theta\left(\beta_{y} \eta_{x}^{\prime}-\alpha_{y} \eta_{x}+h \eta_{x} \beta_{y} \tan \theta\right)+\beta_{y} h \eta_{x}^{\prime}}{-4 \pi} .
\end{aligned}
$$

The parameters $\alpha, \beta$, and $\eta$ are the twiss functions and the horizontal dispersion function. Subscripts 1 and 2 denote dipole entrance and exit, respectively. The first term in the equations corresponds to the first order focusing/defocusing effects of the dipole fringe field and the others are second order terms. Because of the symmetric structure of the lattice, only one dipole has to be evaluated. Moreover, either the entrance or the exit of the dipoles is next to a dispersion free section, so for one of the two fringe field regions, the second order terms have no effect. Suppose that the entrance of the dipole is dispersion free and the bending angle of the dipole is $90^{\circ}$; the dispersion at the exit can be calculated by the transfer matrices for the dipole and edge field:

$$
\begin{aligned}
{\left[\begin{array}{c}
\eta_{x} \\
\eta_{x}^{\prime} \\
1
\end{array}\right]=} & {\left[\begin{array}{ccc}
1 & 0 & 0 \\
h \tan \theta & 1 & 0 \\
0 & 0 & 1
\end{array}\right]\left[\begin{array}{ccc}
0 & \rho & \rho \\
-h & 0 & 1 \\
0 & 0 & 1
\end{array}\right]\left[\begin{array}{l}
0 \\
0 \\
1
\end{array}\right] } \\
= & {\left[\begin{array}{c}
\rho \\
\tan \theta+1 \\
1
\end{array}\right] . }
\end{aligned}
$$



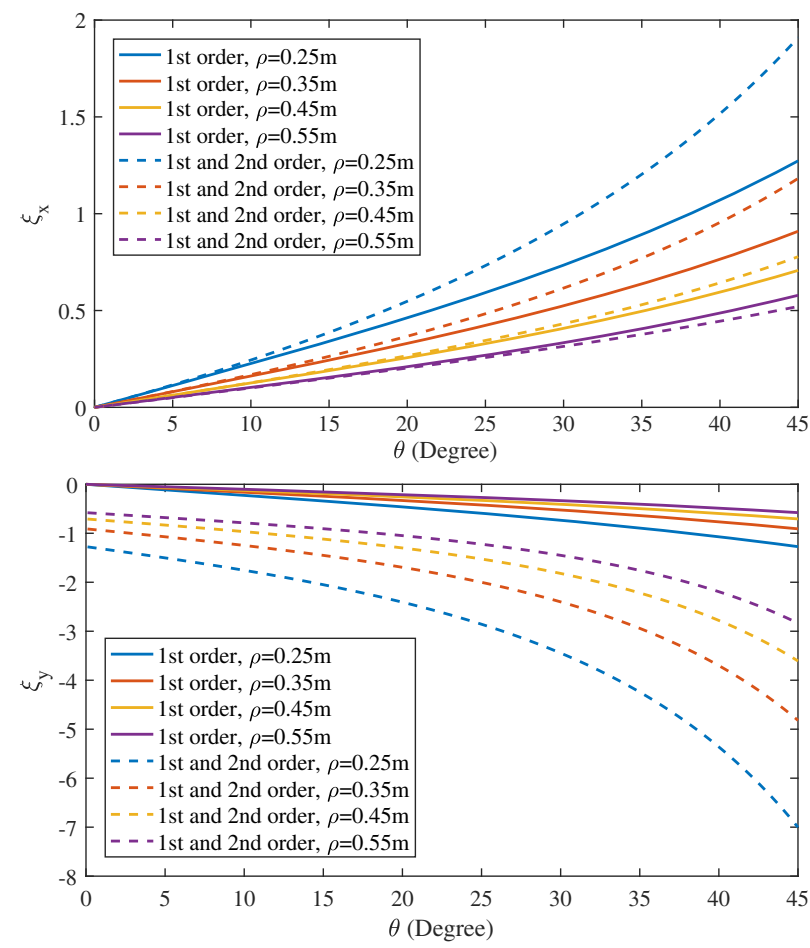

FIG. 2. Relation between the edge angle and chromaticity assuming $\beta_{x, y}=1 \mathrm{~m}$ and $\alpha_{x, y}=1$. The chromaticity is multiplied by 4 to account for the 4 edges located at nonzero dispersion sections.

Substitute Eq. (2) into Eq. (1) and the relation between edge angle and chromaticity is found. The relation between edge angle and chromaticity induced by the dipole fringe field is plotted in Fig. 2, assuming $\beta_{x, y}=1 \mathrm{~m}$ and $\alpha_{x, y}=1$, which are typical values for a storage ring of this size and close to the final optimized values. As can be seen in the figure, vertical chromaticity is more dependent on the edge angle and bending radius than horizontal chromaticity when the second order terms are taken into account. With zero edge angle, the horizontal chromaticity becomes zero and vertical chromaticity remains finite, so however we choose the parameters, the edge field always contributes to vertical chromaticity. The horizontal chromaticity is positive because the edge field is defocusing in the horizontal plane. Natural chromaticity caused by quadrupole error is always negative, so it is beneficial for the edge angle to be nonzero, to help reduce total horizontal chromaticity. Although the vertical chromaticity by the dipole edge field is negative, it does not change much when the edge angle varies from 0 to 25 . Therefore, it is possible to have a nonzero edge angle while keeping vertical chromaticity reasonably small. In our design, the arc length of the bending magnets is chosen to be $0.52 \mathrm{~m}$, corresponding to the bending radius of $0.33 \mathrm{~m}$. The vertical chromaticity is estimated to be about -2 with an edge angle smaller than 23 , which can be further adjusted with quadrupole magnets by changing twiss functions.
The straight sections where the quadrupole magnets are located are $0.8 \mathrm{~m}$ long. The distance between the centers of the quadrupole magnets is $0.175 \mathrm{~m}$. Using thin lens approximation, the transfer matrix for the dispersion function can be written as

$$
\begin{array}{rlrl}
M & =M_{D 1} M_{Q 2} M_{D 2} M_{Q 1} M_{D 2} M_{Q 2} M_{D 1}, \\
M_{D 1} & =\left[\begin{array}{ccc}
1 & L_{1} & 0 \\
0 & 1 & 0 \\
0 & 0 & 1
\end{array}\right], & M_{D 2}=\left[\begin{array}{ccc}
1 & L_{2} & 0 \\
0 & 1 & 0 \\
0 & 0 & 1
\end{array}\right], \\
M_{Q 1} & =\left[\begin{array}{ccc}
1 & 0 & 0 \\
-\frac{1}{f_{1}} & 1 & 0 \\
0 & 0 & 1
\end{array}\right], & M_{Q 2}=\left[\begin{array}{ccc}
1 & 0 & 0 \\
\frac{1}{f_{2}} & 1 & 0 \\
0 & 0 & 1
\end{array}\right],
\end{array}
$$

where $f_{1,2}$ is the quadrupole focal length. To form a DBA, the transfer matrix should be such that the dispersion functions at the beginning and the end of the straight section are equal, while their derivatives are opposite in sign:

$$
\left[\begin{array}{c}
\rho \\
-\tan \theta-1 \\
1
\end{array}\right]=M\left[\begin{array}{c}
\rho \\
\tan \theta+1 \\
1
\end{array}\right] .
$$

Hence, with these constraints, the quadrupole focal length can be numerically solved for a given edge angle:

$$
f_{2}=\frac{0.225\left(-0.0875+f_{1}\right)(2.4713+\tan \theta)}{-0.36552-0.2 \tan \theta+f_{1}(1+\tan \theta)} .
$$

The relation above puts a very strong constraint on the optical functions. There are only two free variables left (i.e., edge angle and $f_{1}$ ) to optimize the optics. The relation between $f_{1}$ and $f_{2}$ is plotted in Fig. 3. As $f_{1}$ approaches $0.0875, f_{2}$ tends to zero, which corresponds to infinitely large quadrupole strength and should be avoided. There is also a singular point where $f_{2}$ tends to infinity, which corresponds to zero quadrupole strength. Equation (3) only

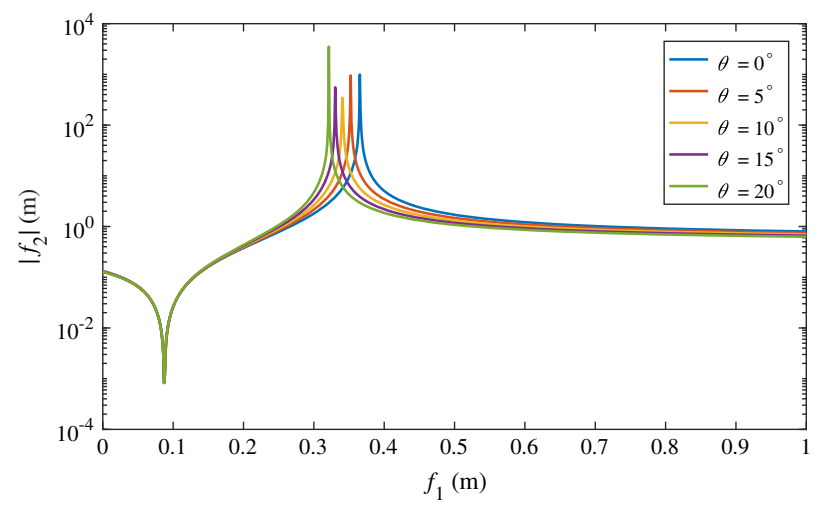

FIG. 3. Relation between $f_{1}$ and $f_{2}$. 

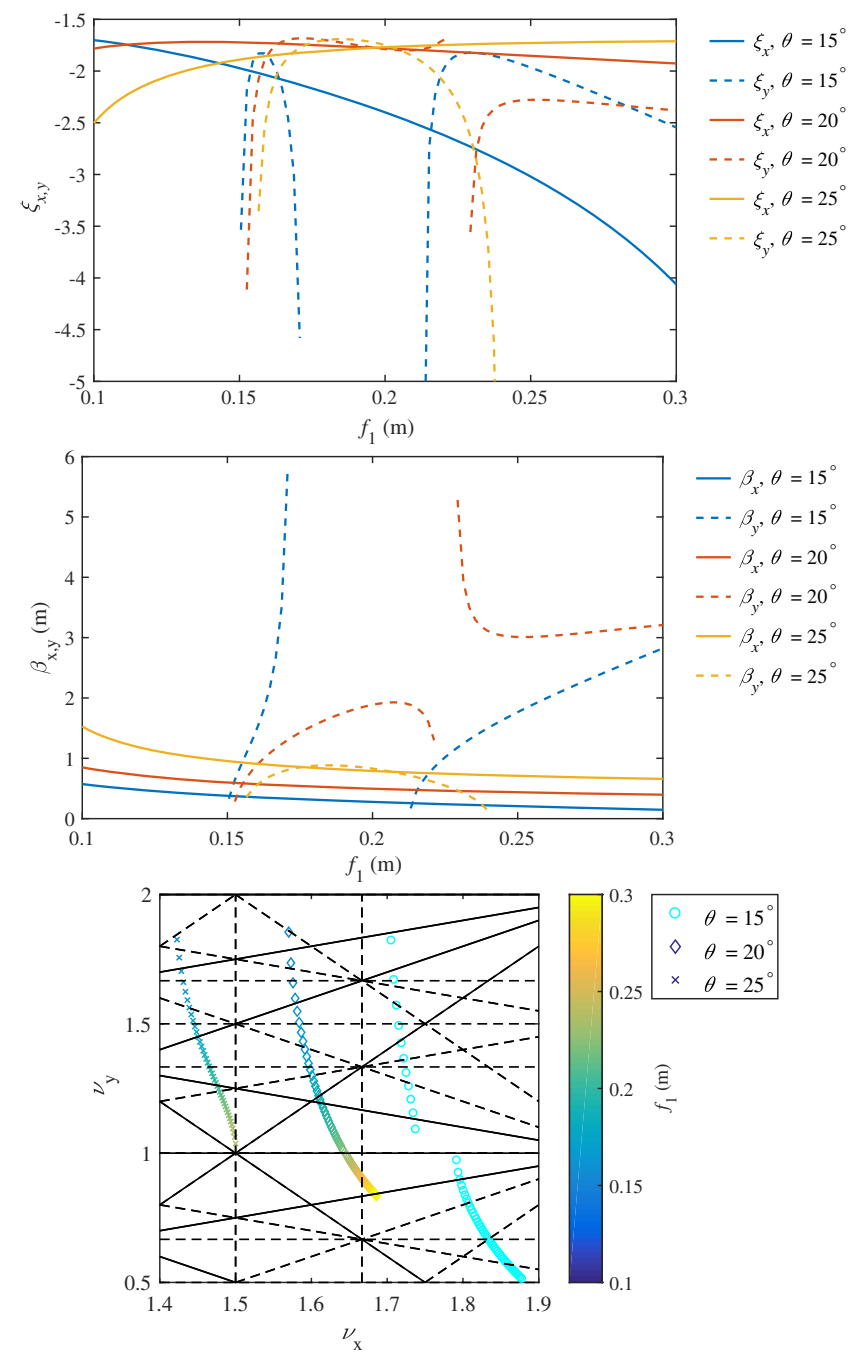

FIG. 4. Chromaticity, betatron functions at IP, and betatron tunes with different quadrupole focal lengths and edge angles.

ensures the lattice cell to be achromatic. To provide proper focusing, the focal length of quadrupole magnets should be reasonably small. Therefore, for later optimization, $f_{1}$ is limited between 0.1 and $0.3 \mathrm{~m}$ (between the two peaks in Fig. 3), so that $f_{2}$ has a similar range from 0.1 to $1 \mathrm{~m}$. On the right side of the second peak, both $f_{1}$ and $f_{2}$ are both too large to provide enough focusing for the lattice to be stable.

To find potential configurations for the lattice, all possible settings of quadrupole focal lengths and dipole edge angles are scanned. Figure 4 shows the scanning results for different edge angles. The lattice is not always stable in the vertical plane when the focal length varies from 0.1 to $0.3 \mathrm{~m}$, as indicated by the gaps in the curves of vertical chromaticity. Hence, the configuration space is further limited. In the cases where the lattice is stable in both planes, horizontal chromaticity is smaller when the edge angle is larger, as indicated by the solid lines in Fig. 4. In order to provide some flexibility, the focal length should be in the flat top area in Fig. 4. Meanwhile, the choice of edge angle and focal length should meet the requirement of small chromaticity. Therefore, the focal length is chosen to be about $0.2 \mathrm{~m}$ and the edge angle between 20 and 25 . The betatron functions at IP shown in Fig. 4 also indicate that these parameters meet the requirement of a small beam size. The tune diagram plotted in Fig. 4 includes resonance lines up to third order. The tunes in both transverse planes have a very limited range when the edge angle and focal length are within the currently optimized parameter range. Horizontal tune has a range from 1.4 to 1.7 , and vertical tune has a range from 1.0 to 1.33 . To avoid systematic resonance lines, the working point is chosen to be near $(1.6,1.1)$.

So far, all of the calculations are done under the thin-lens approximation. For more accurate results, the quadrupoles should be transformed into thick lenses. The lengths of the quadrupoles are chosen such that the tune shifts with amplitude induced by quadrupole fringe fields can contribute to Landau damping. In our design, only the ideal hard edge fringe field is taken in account, because the fringe field profile is unknown prior to measurement and the effect of the hard edge fringe field is the dominant nonlinear effect. The amplitude dependent tune shifts of both transverse planes are proportional to $K_{1}^{2} L$ and dependent on twiss functions [4]:

$$
\begin{aligned}
& \Delta \nu_{x}=\frac{1}{8 \pi} \sum K_{1}^{2} L\left(\beta_{x}^{2} I_{x}+2 \beta_{x} \beta_{y} I_{y}\right), \\
& \Delta \nu_{y}=\frac{1}{8 \pi} \sum K_{1}^{2} L\left(\beta_{y}^{2} I_{y}+2 \beta_{x} \beta_{y} I_{y}\right),
\end{aligned}
$$

where the sums are over all quadrupoles. $I_{x}$ and $I_{y}$ are the actions of the transverse motion.

During the previous optimization process, the integrated normalized quadrupole strength $K_{1} L=1 / f_{1}$ is found to be about $5 \mathrm{~m}^{-1}$ for horizontal focusing quadrupoles and $-2 \mathrm{~m}^{-1}$ for vertical focusing quadrupoles. Assuming that $\beta_{x, y}=1 \mathrm{~m}$ and $I_{x, y}=200 \mathrm{~nm}$, for quadrupoles with a length of $50 \mathrm{~mm}$, the rms tune spreads are about $3 \mathrm{e}-5$, which roughly translates to $0.1 \mathrm{~ms}$ Landau damping time. Hence, in our design, the quadrupoles are all $50 \mathrm{~mm}$ long.

For further optimization and to transform the lattice elements from thin lenses to thick lenses, the simplex algorithm is exploited. The resulting lattice layout is shown in Fig. 5, along with betatron amplitude functions and horizontal dispersion function. The main parameters of the lattice are shown in Table I. The calculation is done with a modified version of ELEGANT [5] and MADX [6]. The parameters calculated by the two codes agree well with each other and with our previous estimation. Some parameters such as detuning parameters are calculated.

Negative chromaticity and the positive momentum compaction factor together may cause head-tail instability. The betatron amplitude functions on both planes are close to each other, so sextupole chromaticity correction is not a 


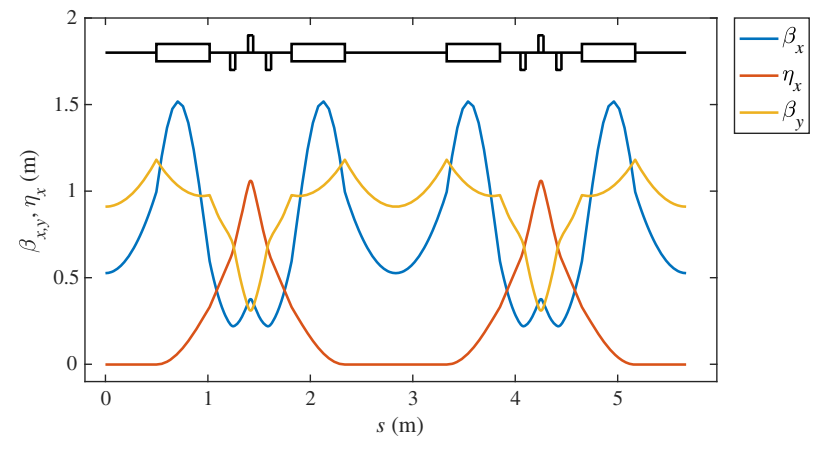

FIG. 5. Layout of the compact electron ring with betatron amplitude functions and horizontal dispersion function.

viable option. It is possible to adjust dispersion function to tune the momentum compaction factor to a negative value by changing quadrupole strengths, but it is preferable that the dispersion functions at the straight sections remain to be zero during operation. To suppress the head-tail instability, we have to rely on Landau damping. Another problem caused by the uncorrected chromaticity is that the tune spread is large, so particles with large momentum deviation may cross dangerous resonance lines and get lost in the process. For a particle with $2 \%$ momentum deviation, both the horizontal and vertical tune shifts are about -0.04 . This may limit the dynamic aperture and thus beam lifetime.

\section{Dynamic aperture}

The dynamic aperture is the stable region of phase space. It can be computed with particle tracking codes. A modified version of ELEGANT is used in the evaluation [5]. To preserve symplecticity and to simulate dipole edge chromatic effects correctly, the Lie map generator by Hwang is used with the Lie exponential map truncated up to eighth order [7].

To evaluate the dynamic aperture, particles with different initial coordinates and momentum deviations are tracked

TABLE I. Lattice parameters.

\begin{tabular}{lcc}
\hline \hline Parameter & ELEGANT & MADX \\
\hline Betatron tunes $\left(\nu_{x} / \nu_{y}\right)$ & $1.605 / 1.063$ & $1.605 / 1.063$ \\
Chromaticities $\left(\xi_{x} / \xi_{y}\right)$ & $-1.918 /-1.939$ & $-1.918 /-2.148$ \\
Chromaticities w/o edge & $-1.871 /-0.476$ & $\ldots$ \\
Second order terms & & 0.1318 \\
Momentum compaction & 0.1318 & 0.8835 \\
Factor $\alpha_{c}$ & & \\
Damping partition & 0.8835 & \\
Number $J_{x}$ & & \\
$\begin{array}{l}\text { Detuning parameters by } \\
\text { Quadrupole fringe }\end{array}$ & $5.39 \mathrm{~m}^{-1} / 13.66 \mathrm{~m}^{-1} / 8.29 \mathrm{~m}^{-1}$ \\
Fields $\left(a_{x x} / a_{x y} / a_{y y}\right)$ & & \\
Radiation damping & \multicolumn{2}{c}{$1.27 \mathrm{~s} / 1.13 \mathrm{~s} / 0.537 \mathrm{~s}$} \\
Time $\left(\tau_{x} / \tau_{y} / \tau_{E}\right)$ & \multicolumn{2}{c}{$1.67 \mathrm{eV}$} \\
Energy loss per turn $\left(U_{0}\right)$ & \multicolumn{2}{l}{} \\
\hline \hline
\end{tabular}

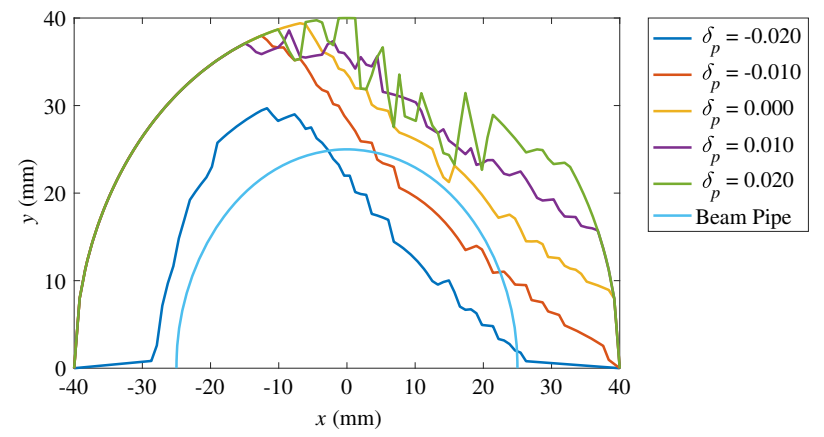

FIG. 6. Dynamic aperture for the norminal lattice.

for many turns to determine the stable region. In our calculation, the dynamic aperture is evaluated at the interaction point and converges after 10000 turns. As is shown in Fig. 6, the minimum dynamic aperture for momentum deviation between $-2 \%$ and $2 \%$ is a bit smaller than the maximum beam pipe radius $25 \mathrm{~mm}$, although it is still over 20 times larger compared to equilibrium beam size, which is about $1 \mathrm{~mm}$.

To gain more insight about the particle diffusion process, a frequency map analysis is performed. Figures 7 and 8 show the tune diffusion rate for particles uniformly distributed in the vacuum chamber and momentum deviation

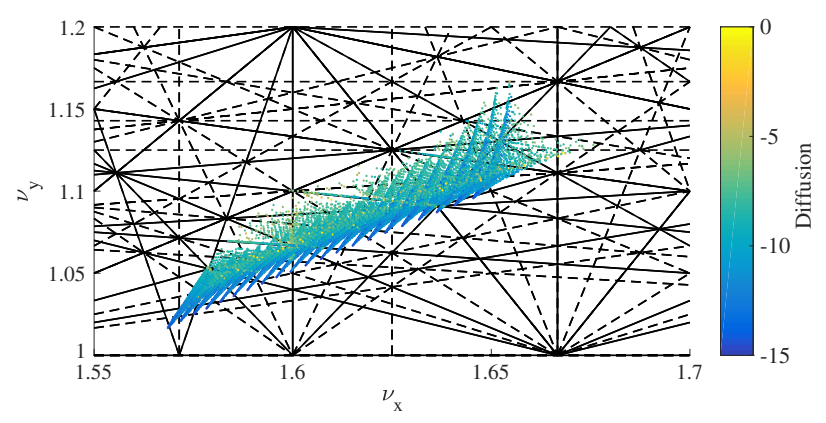

FIG. 7. Tune footprint for off momentum particles.
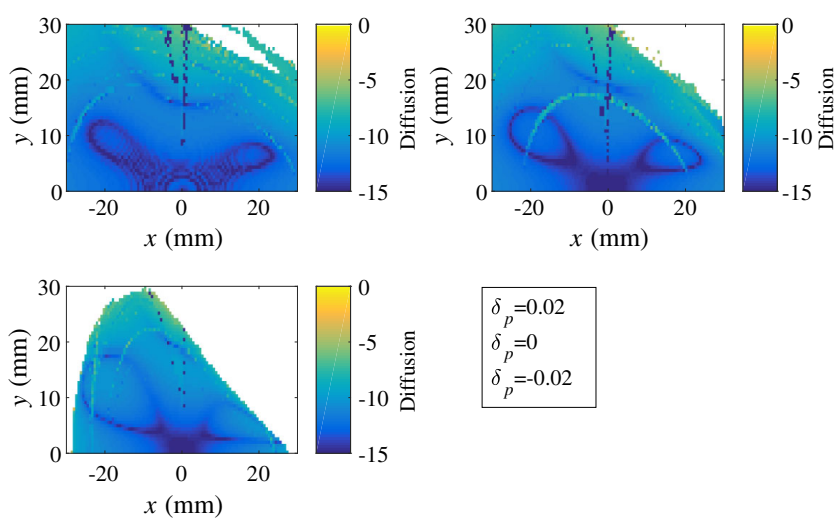

FIG. 8. Diffusion rate of particles distributed uniformly in the beam pipe. 
within $\pm 2 \%$. The diffusion rate is indicated by color, which is defined to be $\log _{10} \sqrt{\Delta \nu_{x}^{2}+\Delta \nu_{y}^{2}}$. As is shown in Figs. 7 and 8 , the tunes are widely spread because of the uncorrected chromaticity. Although some particles are under the influence of high order resonance lines, the diffusion rate for most of the particles in the central region of the vacuum chamber is low. For a beam with large emittances $\epsilon_{x, y}=5 \mu \mathrm{m}$, the transverse rms beam sizes are $1.5 \mathrm{~mm}$ horizontally and $1.7 \mathrm{~mm}$ vertically, so most of the particles are expected to be stable.

\section{BEAM DYNAMICS}

The beam evolution in TTX-II consists of three phases divided according to the dominating effects.

In the first phase, the beam dilutes in phase space due to mismatching. The injection system utilizes the fringe field of bending magnets, which is highly nonlinear. After the beam travels through the fringe field, the beam distribution in phase space is no longer an ellipse and cannot be described accurately with twiss parameters. Matching the beam to lattice twiss parameters results in a mismatch nevertheless. The first phase lasts no more than 10000 turns before the beam reaches a matched state.

In the second phase, collective effects, especially headtail instability, takes control. Because of the uncorrected chromaticity, the lowest mode (mode 0) may be excited and results in the beam centroid oscillation. In TTX-II, the nonlinearity of quadrupoles and dipoles induces a tune spread large enough for Landau damping to take effect. The head-tail growth rate is typically a few milliseconds.

In the last phase, intrabeam scattering, synchrotron radiation, Thomson scattering, and residual gas scattering dominate. Owing to the beam energy, synchrotron radiation damping is weak. As a result, the emittance of the beam grows continuously until the growth rates of the various effects are lower than the radiation damping rate and the beam reaches equilibrium. This phase lasts a few seconds, which equals tens of millions of turns.

\section{A. Injection}

The injection and extraction in this ring is a problem due to its difficulty of the realization of conventional methods, i.e., by means of septum magnets. In our design, the beam is injected and extracted through the fringe fields of bending magnets. A $500 \mathrm{~mm}$ long stripline kicker is located at one of the straight sections to provide a horizontal kick of $0.055 \mathrm{rad}$. The distance between the two electrodes of the kicker is $36 \mathrm{~mm}$ and the voltage on each electrode is $50 \mathrm{kV}$. The maximum repetition frequency of the pulse generator is $10 \mathrm{~Hz}$ to match the repetition frequency of the injector. The existing linear accelerator TTX along with a transfer line serves as the injector for the ring [1]. Because of the symmetric structure of the ring, injection and extraction are the inverse process of each

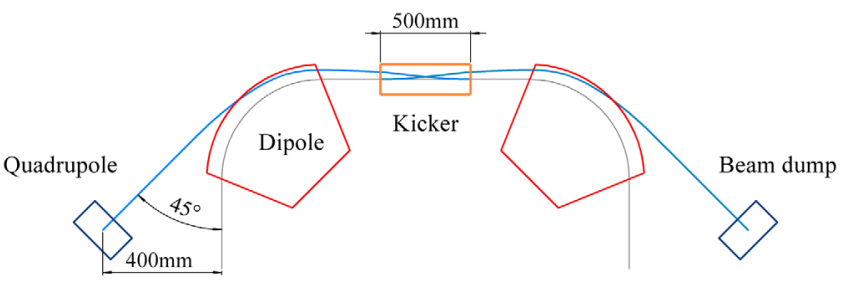

FIG. 9. Injection and extraction scheme.

other. Figure 9 shows the beam injection and extraction scheme. Figure 10 shows the beam trajectory during injection and extraction. The dipole field map is calculated with CST EM Studio [8].

To determine the twiss parameters for the injected beam, the extraction process is first simulated. A matched beam is tracked through the kicker, the fringe field, and then reaches beam dump. As shown in the top right subplot of Fig. 12, before the beam enters the kicker, the horizontal emittance is $20 \mathrm{~nm}$ and its distribution is elliptical When the beam reaches the beam dump, its distribution in phase space is stretched and the emittance grows to $58.5 \mathrm{~nm}$ (top left subplot of Fig. 12). This is because the fringe field of dipole magnets is nonlinear. The effective twiss parameters then can be calculated from the phase space coordinates. The twiss parameters at beam dump and the exit of the injector are the same except that the signs of the alpha functions are opposite. The quadrupole magnets in the linac and the transfer line are adjusted accordingly for matching. The layout of the injector and the twiss functions are shown in Fig. 11.

Injection simulation is done with AsTRA [9]. A beam is tracked from the cathode to the end of the linac, with space charge taken into account, to generate a more realistic beam distribution. The distribution in phase space before the beam enters the fringe field of the dipole magnet is shown in the bottom left subplot of Fig. 12. Again, due to the nonlinearity of the fringe field, the beam dilutes after injection and the emittance grows to $29.6 \mathrm{~nm}$. Moreover, the two subplots on the right side of Fig. 12 are vastly different, indicating that the injected beam is mismatched. This is somewhat unavoidable, because the effective twiss

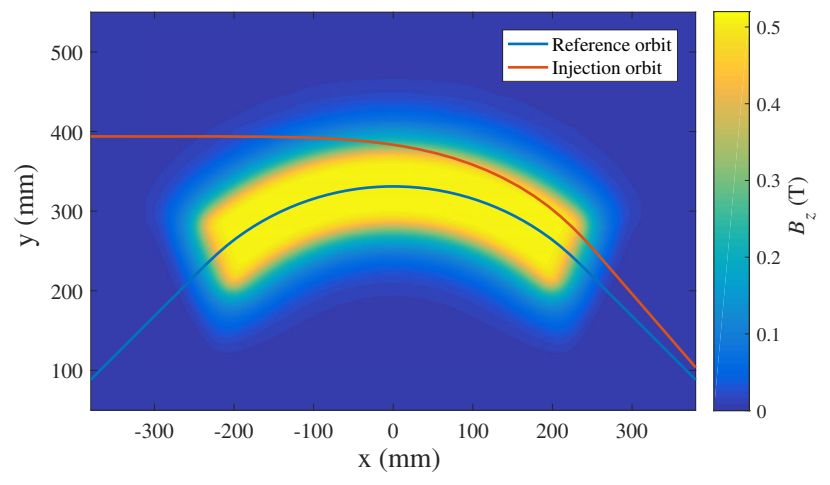

FIG. 10. Simulated beam trajectories. 


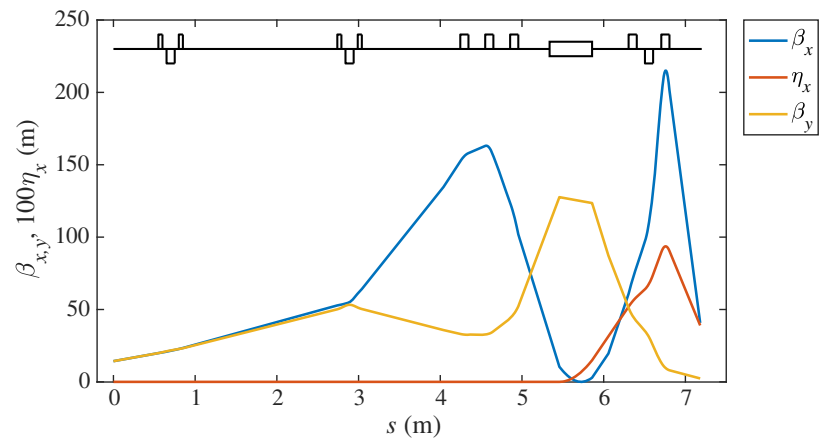

FIG. 11. Layout of the linac and transfer line along with twiss functions and dispersion function. The start of the beamline corresponds to the exit of the accelerating tube.
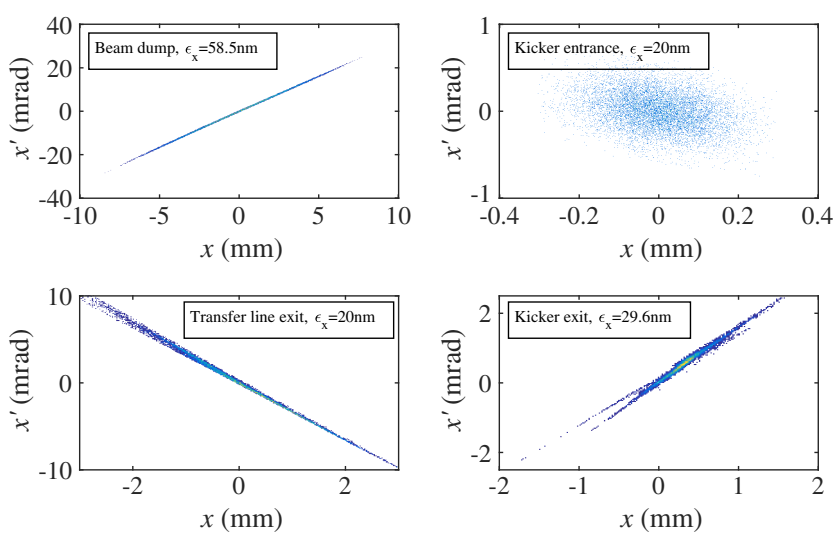

FIG. 12. Horizontal phase space at different locations.

parameters calculated from the phase space coordinates are under the assumption of an elliptical distribution, which is not true when the beam travels through nonlinear fields. However, equilibrium parameters in the storage ring are independent of initial parameters, so in the long run, the mismatching is not important. Moreover, a relatively large emittance contributes to Landau damping and is actually good for beam stability.

Because of the mismatched injected beam, the horizontal emittance undergoes dilution in the first 20000 turns. Emittance converges at $370 \mathrm{~nm}$, as is shown in Fig. 13. Afterwards, the intrabeam scattering (IBS) effect becomes dominant and emittance continues to grow but at a much slower rate. The ring has two operation modes: pulsed mode and steady mode. In pulsed mode, the kicker operates at a frequency of $10 \mathrm{~Hz}$; each time when a new bunch arrives and is to be injected, the old one gets extracted simultaneously. Because of the emittance growth and IBS effect, the x-ray photon flux degrades continually. In steady mode, the electron beam reaches equilibrium before $\mathrm{x}$-ray generation, so the $\mathrm{x}$-ray photon flux remains constant during operation.

There is also a similar mismatching situation in the longitudinal direction. Generally, to achieve high injection
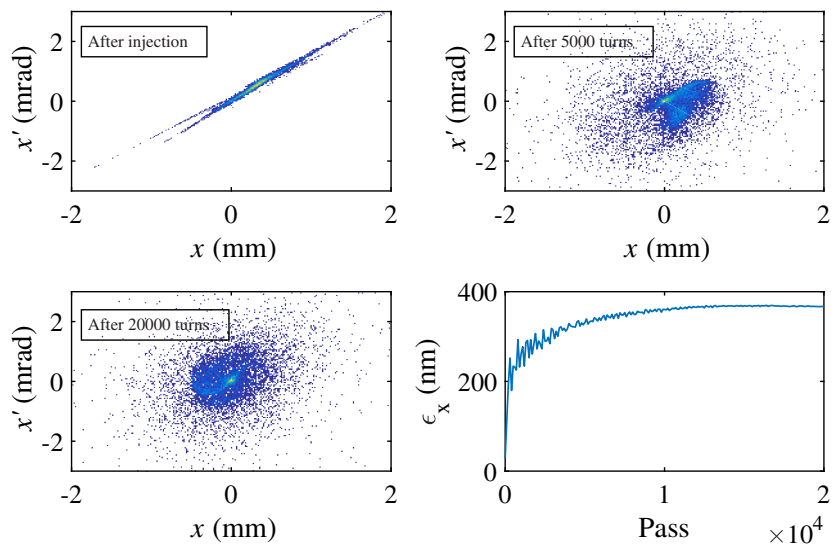

FIG. 13. Emittance increase after injection.
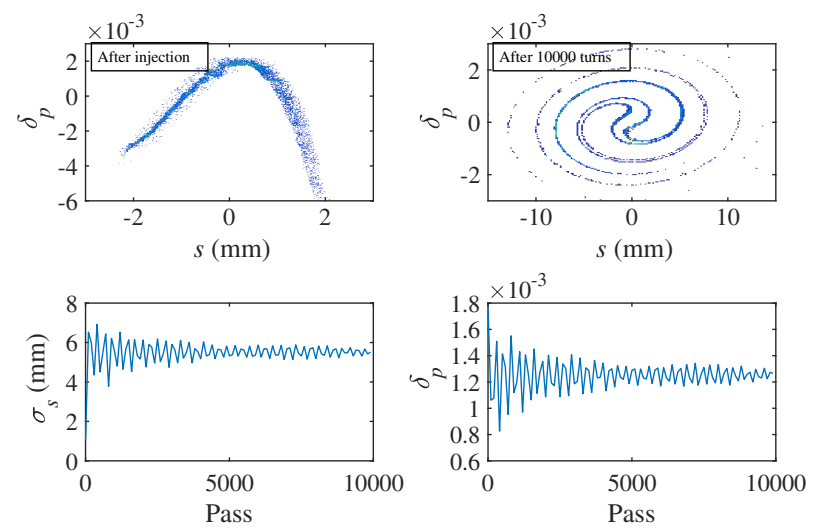

FIG. 14. Longitudinal phase space evolution. Beam reaches pseudomatched state after 10000 turns.

efficiency, the energy spread of the injected beam should be small. To achieve high accelerating efficiency and small energy spread in a linear accelerator, the phase of the accelerating tube is placed around the phase of maximum energy gain. As a result, particles at the head and tail of the bunch gain less energy than those in the center. This results in the curvy shape of longitudinal distribution shown in Fig. 14. Hence, the longitudinal phase distribution of the injected beam is mismatched with the rf bucket. Similar to the transverse case, the beam dilutes in the first 10000 turns after injection before reaching the matched state. The energy spread and bunch length change from $1.8 \%$ and $1.1 \mathrm{~mm}$ to $1.2 \%$ and $5.5 \mathrm{~mm}$, respectively. Table II summarizes the $\mathrm{rf}$ parameters. The frequency of the rf cavity is chosen to be

TABLE II. Radio-frequency parameters.

\begin{tabular}{lc}
\hline \hline Parameter & Value \\
\hline Frequency & $1.428 \mathrm{GHz}$ \\
Voltage & $60 \mathrm{kV}$ \\
Shunt impedance & $4 \mathrm{M} \Omega$ \\
Energy acceptance & $1.5 \%$ \\
\hline \hline
\end{tabular}


$1.428 \mathrm{GHz}$ to match synchronizing system frequency (2856 MHz). The choice of rf voltage has taken several factors into consideration, including energy acceptance and beam lifetime.

\section{B. Head-tail instability}

The head-tail instability of bunched beams was observed and theoretically described many years ago $[10,11]$. Short range transverse wake fields excited by particles at the head of the bunch may excite oscillations at its tail. The particles at the tail are brought to the head by synchrotron motion and continue to excite particles behind. With nonzero chromaticity, the oscillations may add in phase and result in instability. For the lowest head-tail mode (mode number $k=0$ ) to be stable, chromaticity and momentum compaction must be of the same sign. In this case, higher modes may be unstable, but their growth rates are usually smaller than that of the lowest mode. The natural chromaticity in storage rings is negative, and in most machines the momentum compaction is positive, so usually the chromaticity is corrected with sextupoles to be positive.

In TTX-II, no chromaticity correction scheme is viable due to space limitation and optical functions. With chromaticity being negative, the only head-tail mode that would be excited is the lowest mode. In this mode, all particles oscillate in phase, which corresponds to a rigid dipole oscillation at the betatron frequency. To suppress this instability, one can rely on an active feedback damper or other damping mechanisms. In our case, the instability can be easily suppressed by Landau damping.

The complex coherent frequency shift of the head-tail mode with mode number $\mathrm{m}$ for bunches with air bag longitudinal distribution can be expressed as [11]

$\Delta \Omega_{m}=-i \frac{N_{b} r_{0} c}{8 \pi^{2} \gamma \nu_{\beta}} \int_{-\infty}^{\infty} Z_{1}^{\perp}(\omega) J_{m}^{2}\left(\frac{\omega \hat{z}}{c}-\frac{\xi \omega_{\beta} \hat{z}}{c \eta}\right) \mathrm{d} \omega$

with $N_{B}$ the number of the particles in the bunch, $\hat{z}$ the amplitude of synchrotron motion, $r_{0}$ classical particle radius, $J_{m}$ the Bessel function, and $\eta$ the slippage factor.

The resistive wall impedance is one of the main contributions to $Z_{1}^{\perp}$, and in this case, the growth rate and coherent tune shift can be more specifically expressed as [12]

$$
\begin{aligned}
& \frac{1}{\tau_{m}}=-\frac{\sqrt{2} J_{m}}{\pi^{2} .5} \frac{r_{0} c}{\sqrt{\mu \sigma_{c}}} \frac{N_{b} \xi \sqrt{\hat{z}}}{\eta \gamma b^{3} \nu_{\beta}}, \\
& \Delta \nu=-\frac{K_{m}}{\sqrt{2} \pi^{2} .5} \frac{r_{0} c}{\sqrt{\mu \sigma_{c}}} \frac{N_{b} \sqrt{\hat{z}}}{\omega_{0} \gamma b^{3} \nu_{\beta}},
\end{aligned}
$$

with $b$ the beam pipe half height, $\mu$ permeability, and $\sigma_{c}$ conductivity. The factors $K_{m}$ and $J_{m}$ are 13.6 and 2.9 for the lowest mode.

Another main source of impedance is geometric impedance induced by discontinuities in vacuum chambers which can be approximated as broadband impedance. The broadband impedance of mode number $\mathrm{m}$ can be described as

$$
\begin{aligned}
& Z_{m}^{\|}=\frac{R_{s}}{1+i Q\left(\frac{\omega_{R}}{\omega}-\frac{\omega}{\omega_{R}}\right)}, \\
& Z_{m}^{\perp}=\frac{2 c}{b^{2 m} \omega} Z_{0}^{\|},
\end{aligned}
$$

where $R_{s}$ is the shunt impedance, $\omega_{R} \approx c / b$ is the resonance frequency, and $Q$ is the quality factor that is usually close to unity. The growth rate and coherent tune shift in this case have no explicit expressions like in the case of resistive wall impedance and can be evaluated numerically with Eq. (7).

Both the resistive wall impedance and broad band impedance here are under the assumption of a round chamber cross section. In TTX-II, the vacuum chambers are rectangular with half heights of $a=25 \mathrm{~mm}$ and $b=13 \mathrm{~mm}$, so Eqs. (6) and (7) are not strictly applicable.

When the chamber cross section is not round, the transverse wake function can be linearized and divided into two parts, including the dipolar part proportional to the coordinate of the source particle $\left(x_{1}\right.$ or $\left.y_{1}\right)$ and the quadrupolar part proportional to the coordinate of the witness particle $(x$ or $y)$ [13]:

$$
W_{\perp}=\left[\begin{array}{l}
W_{1, x} x_{1}+W_{2} x \\
W_{1, y} y_{1}-W_{2} y
\end{array}\right]
$$

The coefficients depend on the ratio of the height to the width of the vacuum chamber. For a rectangular chamber, the coefficients in Eq. (8) can be numerically calculated. The coefficients as functions of the ratio of chamber height to chamber width is plotted in Fig. 15. The coefficients are normalized with $W_{1}^{\text {round }}$ which is the wake function coefficient when the chamber is round and the radius is $b$.

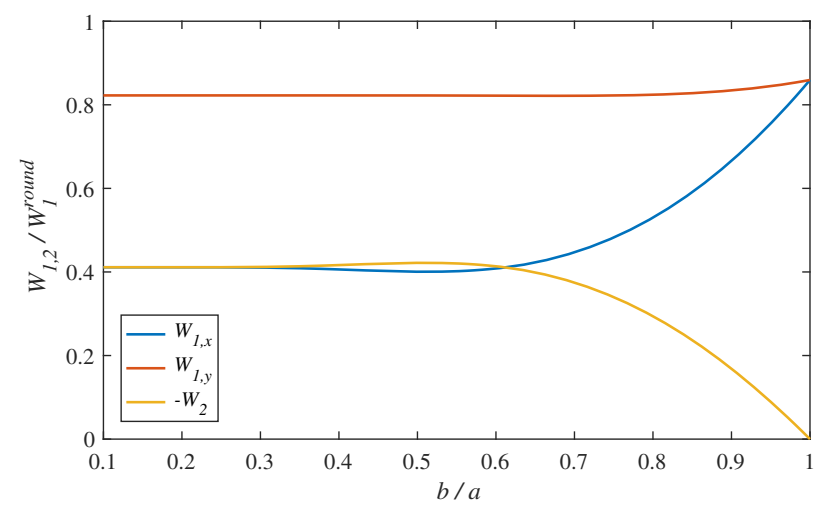

FIG. 15. Coefficients of the wake function versus the ratio of chamber height to chamber width. The coefficients are normalized with $W_{1}^{\text {round }}$, the wake function when chamber is round and the radius equals $b$. 
The quadrupolar wakefield introduces incoherent tune shifts to the particles, while the dipolar wakefield introduces coherent tune shift and may excite beam centroid motion. In TTX-II, only the lowest mode $(m=0)$ of head-tail instability can be excited, while all the other higher order modes are exponentially damped due to the different signs of chromaticity and momentum compaction. In the lowest mode, all particles oscillate in phase, so it can be assumed that the witness particles have the same transverse displacement as the source particles. In this scenario, the complex coherent frequency shift of head-tail instability can be estimated by Eq. (6) to Eq. (8) with some modification:

$$
\begin{aligned}
& \operatorname{Re}\left(\Delta \Omega_{0, x}\right)=\frac{W_{1, x}+W_{2}}{W_{1}^{\text {round }}} \operatorname{Re}\left(\Delta \Omega_{0}^{\text {round }}\right), \\
& \operatorname{Im}\left(\Delta \Omega_{0, x}\right)=\frac{W_{1, x}}{W_{1}^{\text {round }} \operatorname{Im}\left(\Delta \Omega_{0}^{\text {round }}\right),} \\
& \operatorname{Re}\left(\Delta \Omega_{0, y}\right)=\frac{W_{1, y}-W_{2}}{W_{1}^{\text {round }}} \operatorname{Re}\left(\Delta \Omega_{0}^{\text {round }}\right), \\
& \operatorname{Im}\left(\Delta \Omega_{0, y}\right)=\frac{W_{1, y}}{W_{1}^{\text {round }} \operatorname{Im}\left(\Delta \Omega_{0}^{\text {round }}\right) .}
\end{aligned}
$$

It's indicated in Fig. 15 that when the chamber half width $a$ is significantly larger than chamber half height $b$ ( $b / a<0.6), W_{1, x}+W_{2}$ tends to zero. As a result, the horizontal frequency shift becomes zero. Hence, in TTX-II, the head-tail instability is more likely to occur in a vertical plane, with $b / a$ being 0.52 . The corresponding coefficients are as follows:

$$
\begin{aligned}
& {\left[\frac{W_{1, x}}{W_{1}^{\text {round }}}\right]_{\frac{b}{a}=0.52}=0.401,\left[\frac{W_{1, y}}{W_{1}^{\text {round }}}\right]_{\frac{b}{a}=0.52}=0.822,} \\
& {\left[\frac{W_{2}}{W_{1}^{\text {round }}}\right]_{\frac{b}{a}=0.52}=-0.422 .}
\end{aligned}
$$

Table. III summarizes the growth rates and real tune shifts for rectangular chamber due to resistive wall impedance (RW) and geometric broad band impedance (BB). The broadband impedance induced by discontinuities in a vacuum chamber is assumed to be $\frac{z_{0}^{\|}}{n}=0.1 \Omega$. The bunch length is $5.5 \mathrm{~mm}$ and the beam pipe is made of aluminium. The beam charge is $1 \mathrm{nC}$.

TABLE III. Growth rate and tune shift of the 0 mode instability.

\begin{tabular}{lcr}
\hline \hline Parameter & RW & \multicolumn{1}{c}{ BB } \\
\hline Growth rate $\left(\frac{1}{\tau_{x} \omega_{0}}\right)$ & $6.24 \mathrm{e}-8$ & $1.16 \mathrm{e}-7$ \\
Betatron tune $\operatorname{shift}\left(\Delta \nu_{x}\right)$ & $9.20 \mathrm{e}-8$ & $5.82 \mathrm{e}-8$ \\
Growth rate $\left(\frac{1}{\tau_{y} \omega_{0}}\right)$ & $1.28 \mathrm{e}-7$ & $2.39 \mathrm{e}-7$ \\
Betatron tune $\operatorname{shift}\left(\Delta \nu_{y}\right)$ & $-5.42 \mathrm{e}-6$ & $-3.43 \mathrm{e}-6$ \\
\hline \hline
\end{tabular}
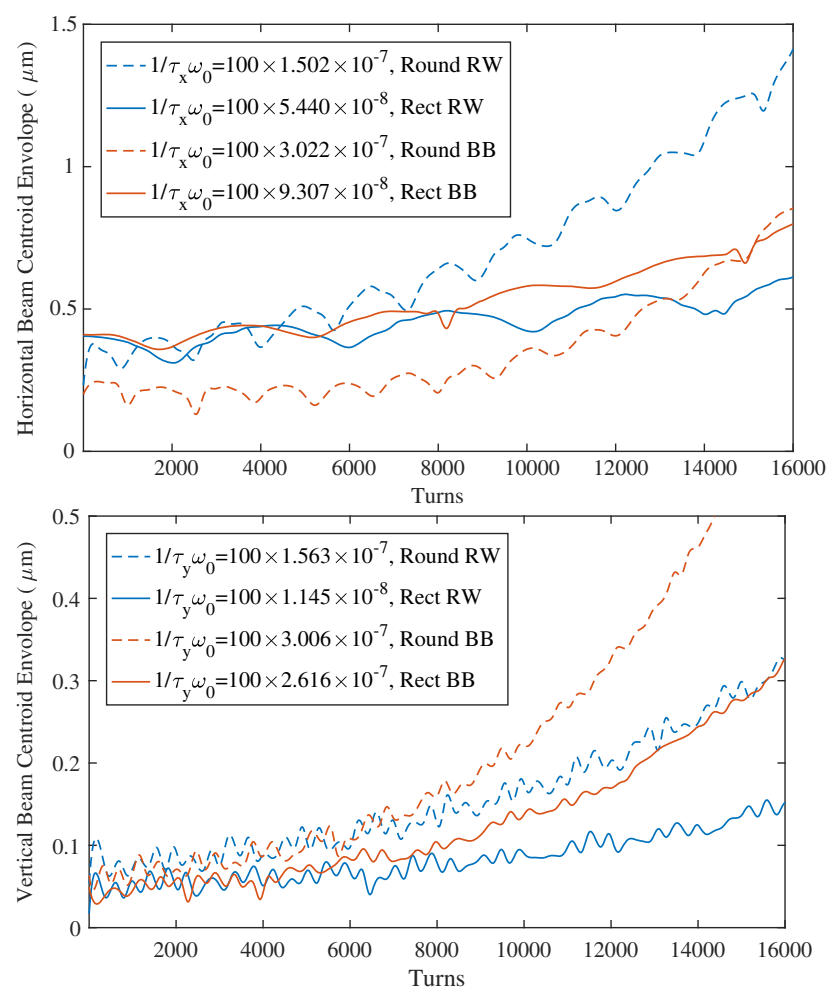

FIG. 16. Envelopes of beam centroid over time with growth rates.

Equation (9) also provides a way to simulate the effects of the rectangular chamber. The verify our calculation, the HEADTAIL code [14] is used for particle tracking, with modification to accept air-bag distribution and total impedance of both resistive wall impedance and geometric impedance. To speed up the calculation, the wakefield kick experienced by the particles are multiplied by a factor of 100 , so the growth rates and tune shifts would be increased by 100 times. The results are shown in Figs. 16 and 17 .

The growth rates and tune shifts in both directions in the case of the rectangular chamber are in accordance with the ones listed in Table. III. The ratios of the growth rates in the case of a round chamber to the case of a rectangular chamber agrees with the coefficients $W_{1, x}$ and $W_{1, y}$ given in Fig. 15 and Eq. (10). The case where only the quadrupolar wakefield is applied to the beam is also calculated, indicated by the dotted lines in Fig. 17. The tune shift due to the quadrupolar wakefield in a rectangular chamber is shown as well. The coherent horizontal tune shift is compensated compared with the case of a round chamber because the quadrupolar wakefield is focusing horizontally and increases betatron tune. In the meantime, the vertical tune shift is enhanced. In conclusion, the results of Eq. (10) and particle tracking agree well with each other.

The air bag model assumes that particles concentrate at the head and tail of the bunch, while in reality, bunches in storage rings usually have Gaussian distribution profiles. As a result, Eq. (5) serves as a rough and likely overestimated 

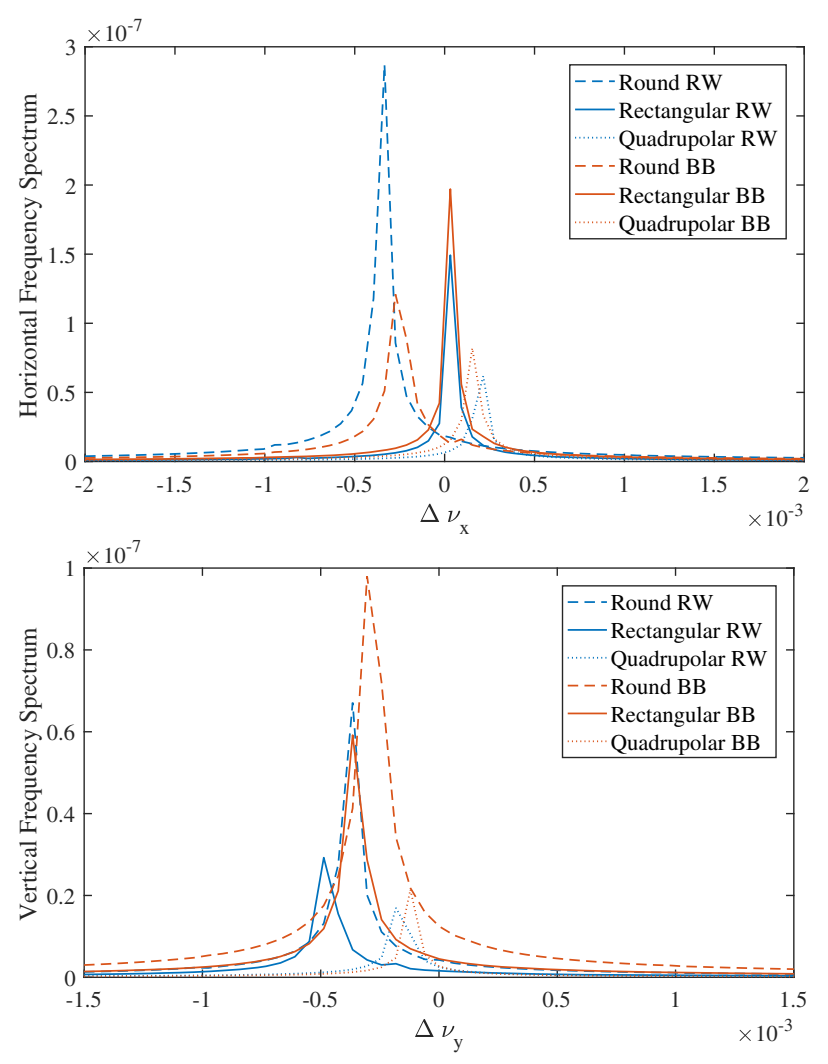

FIG. 17. Frequency spectrums of beam centroid motion. The tune shift due to the quadrupolar wakefield in rectangular chamber is shown as well.

approximation of the growth rate and tune shift.To suppress the instability by Landau damping, the incoherent tune spread must be larger than both the growth rate and the tune shift. Table IV lists the detuning needed for stabilizing the instability. The detuning parameters are calculated under the assumptions that horizontal and vertical emittances are 370 and $20 \mathrm{~nm}$, respectively, and that the beam distribution is Gaussian. For example, in the horizontal plane, the detuning parameter $\alpha_{x x}=0.482 \mathrm{~m}^{-1}$ corresponds to an average horizontal tune shift of $1.78 \mathrm{e}-7$, which is larger than the total head-tail growth rate and tune shift combining resistive wall impedance and geometric impedance. Alternately, the coupling detuning parameter $\alpha_{x y}=8.92 \mathrm{~m}^{-1}$ can provide the same average tune shift.

The detuning in TTX-II mainly comes from the fringe field of dipoles and quadrupoles, and can be increased by adding octupole fields (by octupole magnets or octupole

TABLE IV. Detuning parameters needed for Landau damping.

\begin{tabular}{lcr}
\hline \hline Detuning parameter & $x$ & $y$ \\
\hline$\alpha_{x x}$ & $0.482 \mathrm{~m}^{-1}$ & $\ldots$ \\
$\alpha_{x y}$ & $8.92 \mathrm{~m}^{-1}$ & $26.6 \mathrm{~m}^{-1}$ \\
$\alpha_{y y}$ & $\cdots$ & $492.5 \mathrm{~m}^{-1}$ \\
\hline \hline
\end{tabular}

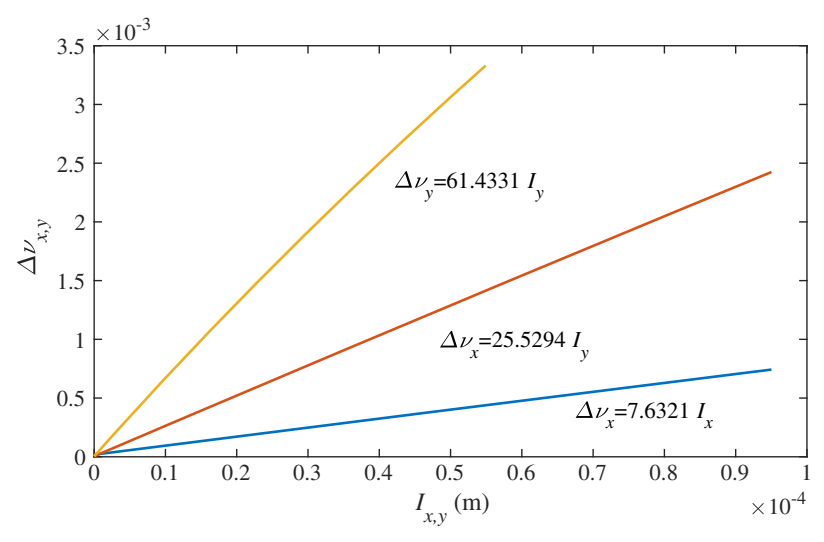

FIG. 18. Tune shifts due to different particle actions.

field components in existing magnets) into the ring. Detuning parameters can be calculated from the one-turn Lie map generator, although it is usually difficult to coalesce all the Hamiltonians of the elements in the ring. An alternate way to calculate the detuning parameters is particle tracking. A group of electrons with different initial transverse coordinates are launched into the ring and tracked for 1024 turns. Afterwards, Fast Fourier transformation is performed to find out the betatron tunes of each particle. Figure 18 shows the fitting results of betatron tunes. The horizontal detuning parameter and coupling detuning parameter are sufficient for horizontal and vertical head-tail instability respectively, according to Table IV.

To verify the instability can be suppressed by Landau damping, particle tracking by the HEADTAIL code is performed. To speed up the calculation, the impedance is increased by a factor of 100 , which results in the growth rates and tune shifts to be increased by a factor of 100 . The detuning parameters are scaled up accordingly.

As is shown in Fig. 19, the motion of the bunch centroid is stabilized with Landau damping. Frequency analysis shown in Fig. 20 further confirms the stability of the beam. Without tune spreads, the head-tail mode is clearly seen in the frequency spectrum. With betatron detuning, the headtail mode is washed out by the tune spread. Both the growth rate and tune shift without tune spread are a bit smaller than those in the case of airbag beam because the particles are more concentrated in the bunch center rather than bunch head and tail.

In conclusion, the mode 0 head-tail instability will not be a problem despite the fact that the natural chromaticity is not corrected. Its growth rate and tune shift are small and can be easily suppressed by the intrinsic tune spread in the ring.

\section{Equilibrium parameters}

In electron storage rings, the equilibrium beam parameters are mainly dependent on synchrotron radiation damping, quantum excitation, residual gas scattering, and intrabeam scattering. In a low energy electron ring, intrabeam scattering is the dominant effect. 

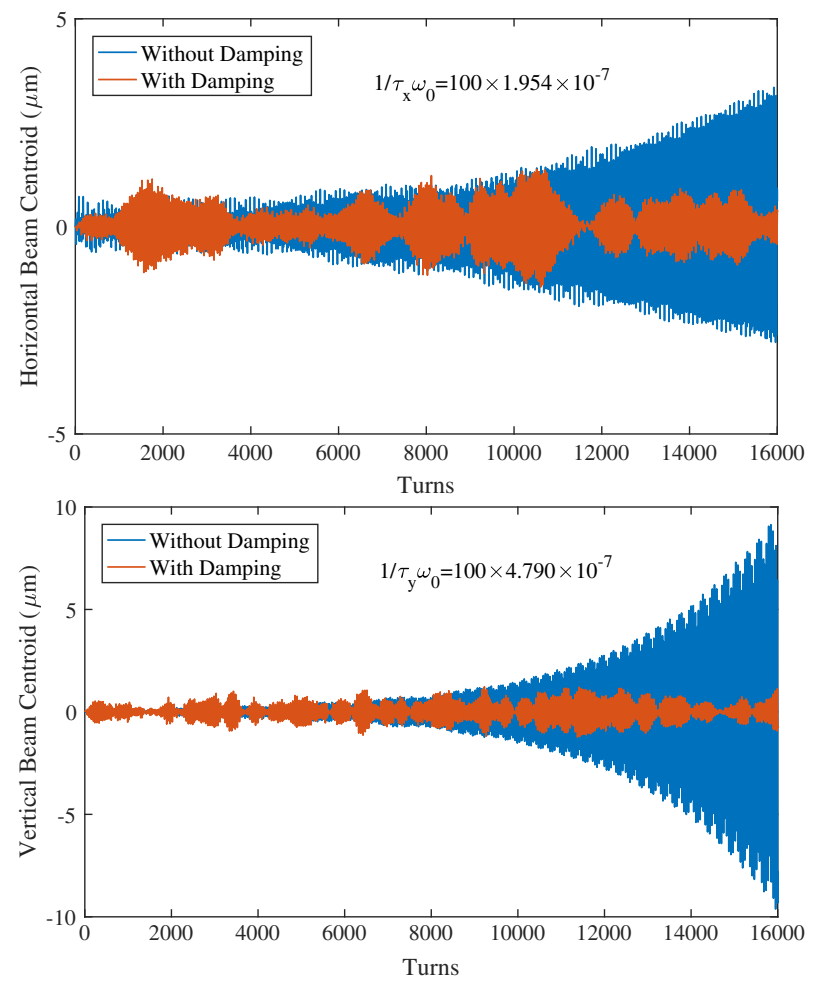

FIG. 19. Motion of beam centroid with and without detuning.
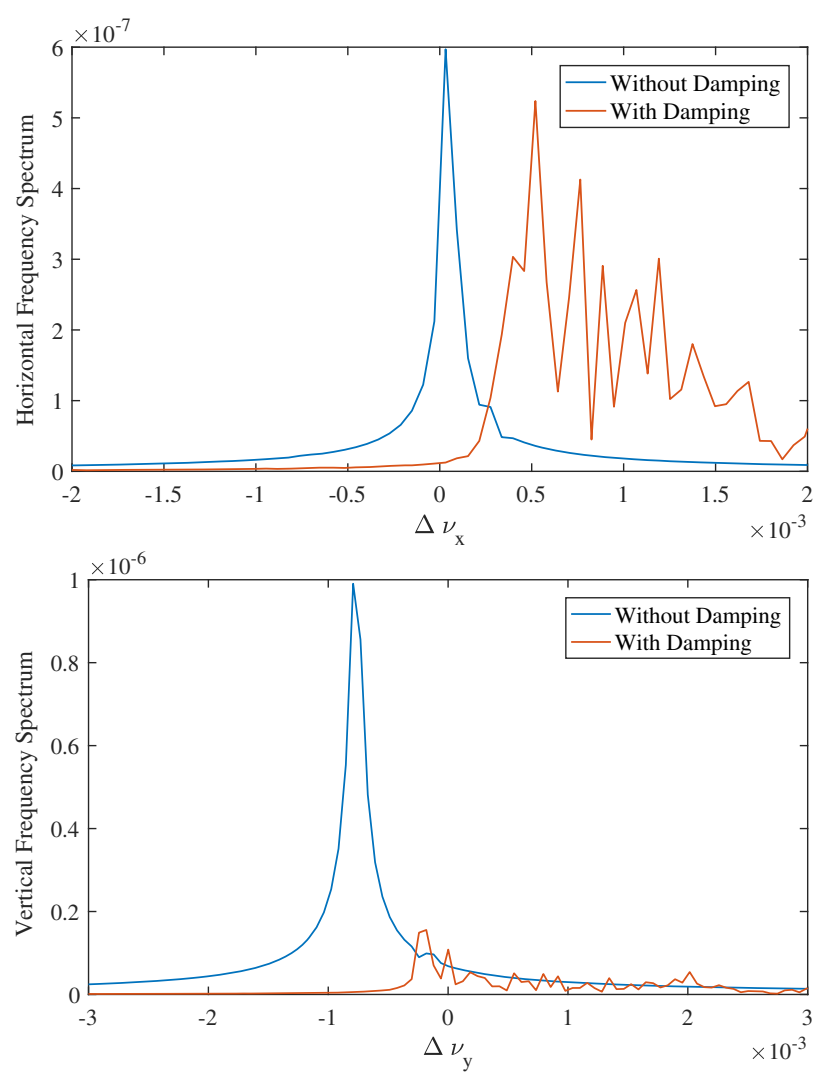

FIG. 20. Frequency spectrum of the beam centroid motion.

After the beam reaches a quasiequilibrium state, the evolution of beam parameters can be calculated via a numerical solution of the following system of equations:

$$
\begin{aligned}
\frac{\mathrm{d} \varepsilon_{x}}{\mathrm{~d} t} & =-\frac{2 \varepsilon_{x}}{\tau_{x}}+\frac{\mathrm{d} \varepsilon_{x, \mathrm{SR}}}{\mathrm{d} t}+\frac{\mathrm{d} \varepsilon_{x, \text { IBS }}}{\mathrm{d} t}+\frac{\mathrm{d} \varepsilon_{x, \mathrm{RGS}}}{\mathrm{d} t}, \\
\frac{\mathrm{d} \varepsilon_{y}}{\mathrm{~d} t} & =-\frac{2 \varepsilon_{y}}{\tau_{y}}+\frac{\mathrm{d} \varepsilon_{y, \mathrm{SR}}}{\mathrm{d} t}+\frac{\mathrm{d} \varepsilon_{y, \mathrm{IBS}}}{\mathrm{d} t}+\frac{\mathrm{d} \varepsilon_{y, \text { RGS }}}{\mathrm{d} t}, \\
\varepsilon_{y} & =\kappa \varepsilon_{x}, \\
\frac{\mathrm{d} \sigma_{E}^{2}}{\mathrm{~d} t} & =-\frac{2 \sigma_{E}^{2}}{\tau_{E}}+\frac{\mathrm{d} \sigma_{E, \mathrm{SR}}^{2}}{\mathrm{~d} t}+\frac{\mathrm{d} \varepsilon_{y, \mathrm{IBS}}}{\mathrm{d} t} .
\end{aligned}
$$

The first terms in the equations correspond to synchrotron radiation damping. The subscripts in other terms indicate emittance growth rate contributed by different sources. $\kappa$ is the emittance coupling factor.

The calculation of radiation damping time and quantum excitation follows conventional formulas [15]. The effect of inverse Compton scattering is approximated as undulator radiation, with undulator wavelength being half the laser wavelength [16], and is included in the synchrotron radiation terms. Table V summarizes the laser cavity parameters and equivalent undulator parameters, with radiation damping time modified to reflect the effects of inverse Compton scattering.

The IBS growth rates are estimated according to Bjorken and Mtingwas's formula [17]. Bjorken and Mtingwas's formula only applies to Gaussian beams. It takes only 0.38 and $0.19 \mathrm{~ms}$ for the injected beam to reach a quasiequilibrium state, which is short compared to radiation damping times, so the quasiequilibrium emittances and energy spread are taken as the initial values for the equations above.

The amplitude of the residual gas scattering effect is proportional to the average of the beta functions, the gas partial pressure, and the radiation length. The residual gas composition varies from case to case and is unknown prior to measurement. In our calculation, the residual gas is assumed to be pure nitrogen. The emittance growth due to residual gas scattering is estimated with Eq. (12), where $P_{g}$ is the pressure

TABLE V. Laser cavity parameters and equivalent undulator parameters with modified damping times.

\begin{tabular}{lc}
\hline \hline Parameters & Value \\
\hline Wavelength $\left(\lambda_{L}\right)$ & $800 \mathrm{~nm}$ \\
Pulse energy $\left(E_{L}\right)$ & $1 \mathrm{~mJ}$ \\
FWHM pulse length $\left(\tau_{L}\right)$ & $100 \mathrm{ps}$ \\
Spot size $\left(\omega_{0}\right)$ & $100 \mu \mathrm{m}$ \\
Undulator length $\left(\lambda_{u}\right)$ & $1.5 \mathrm{~mm}$ \\
Pole number & 7500 \\
Undulator parameter $\left(K_{u}\right)$ & $5.26 \mathrm{e}-4$ \\
Radiation damping length $\left(\tau_{x} / \tau_{y} / \tau_{E}\right)$ & $0.965 \mathrm{~s} / 0.883 \mathrm{~s} / 0.424 \mathrm{~s}$ \\
Energy loss per turn $\left(U_{0}\right)$ & $2.14 \mathrm{eV}$ \\
\hline \hline
\end{tabular}



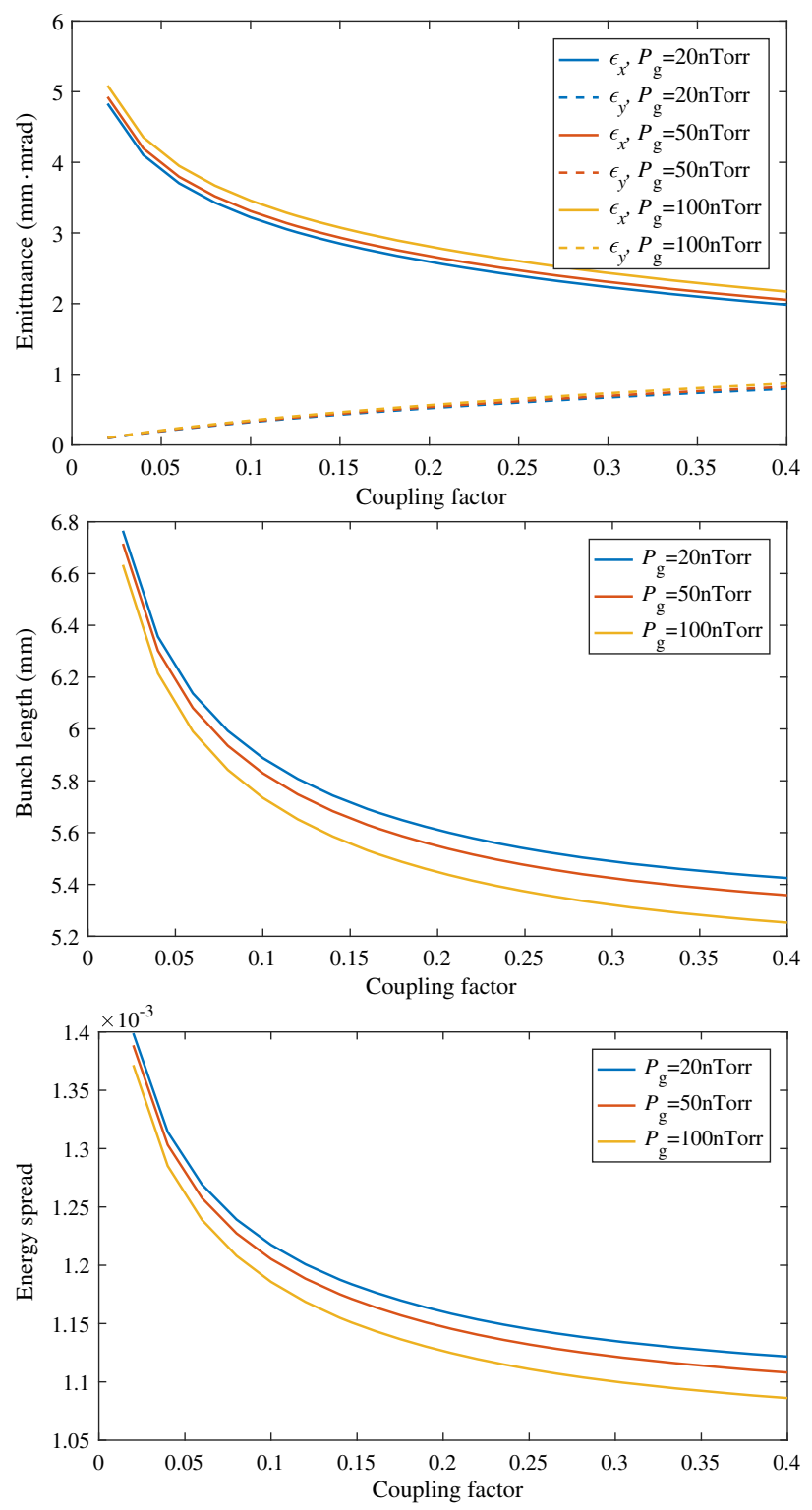

FIG. 21. Equilibrium parameters with varying gas pressure and coupling factor.

of a gas at room temperature, $A_{g}$ is the gram molecular weight of a gas, and $X_{0 g}$ is the radiation length [18],

$$
\frac{1}{\tau_{R} G S}=2.345 \frac{\gamma\left\langle\beta_{x, y}\right\rangle}{\beta \varepsilon_{N}}\left(\frac{e}{p c}\right)^{2} \frac{P_{g} A_{g}}{X_{0 g}} .
$$

The equilibrium parameters under different pressure and coupling factors are plotted in Fig. 21. Gas pressure plays a small part in the evolution of beam parameters, so it is assumed to be 20 nTorr in the following context. The variation of bunch length and energy spread are within $20 \%$ when the coupling factor varies from 0.02 to 0.4 . Meanwhile, both horizontal and vertical emittances are more affected by the coupling factor.

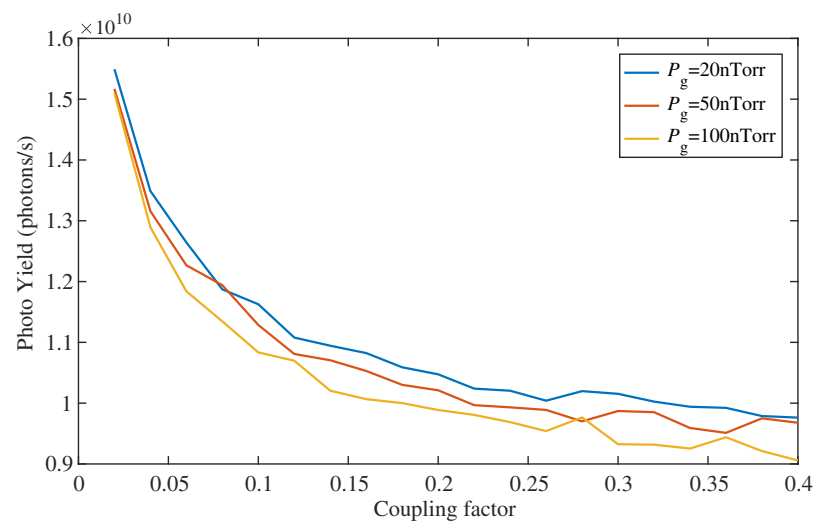

FIG. 22. Photon yield with varying gas pressure and coupling factor.

When the electron beam interacts with the laser beam, the number of backscattered photons are dependent on electron bunch intensity and laser power density. As the coupling factor increases, both bunch length and horizontal beam size decrease, while vertical beam size increases. It is shown in Fig. 22 that vertical beam size is dominant over photon yield, since photon yield decreases with increasing coupling factor. The average photon yield is about $10^{10}$ photons per second for coupling factor varying from 0.02 to 0.4 .

\section{Beam lifetime}

The beam lifetime in TTX-II is limited by the Touschek effect, which describes the momentum transfer from the transverse into the longitudinal direction due to binary collisions between electrons. When the momentum deviation of the collided electrons due to Touschek effect exceeds the momentum aperture of the ring, the electrons will get loss. In the long run, the brightness of the electron beam will slowly degrade, leading to photon yield decrease.

Piwinski [19] derived a general formula for calculating Touschek lifetime of a stored beam for arbitrary ratios of beam height to beam width and arbitrary energies in the rest frame of the colliding particles. The beam lifetime is dependent on beam dimensions, beam population beam energy, and momentum aperture. Beam dimensions are directly related to emittances, which is calculated in the previous section. Momentum aperture is limited by $\mathrm{rf}$ bucket height, transverse dynamics, and physical aperture.

To determine momentum aperture, particles are tracked starting from the exit of each element and the momentum deviation of the particles is increased gradually. If the particle gets lost, the corresponding momentum deviation is considered maximum stable momentum deviation. The momentum aperture calculated in this way is plotted in Fig. 23. Because of large dispersion at the quadrupole magnets, horizontal betatron motion amplitude is very sensitive to momentum change. When two electrons exchange energy with each other, the increase in betatron 


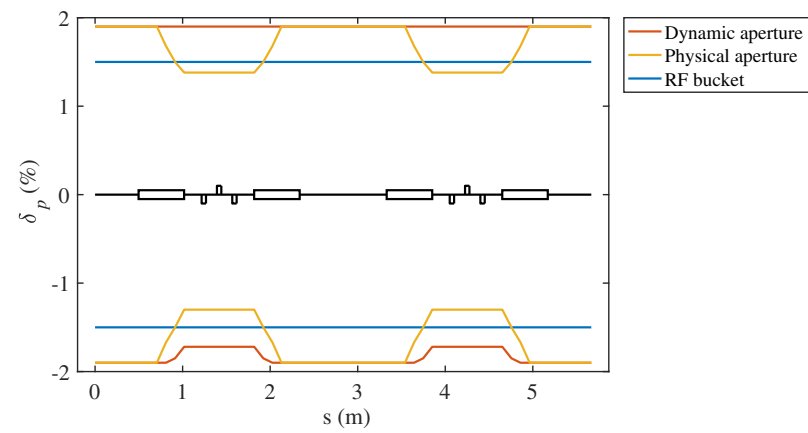

FIG. 23. Momentum aperture limited by different source.

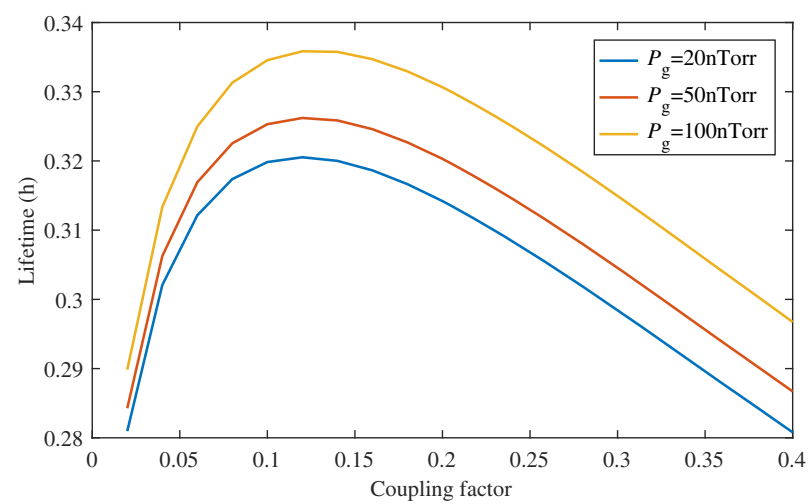

FIG. 24. Touschek lifetime under different gas pressure and varying coupling factor.

motion amplitude is proportional to the momentum change and dispersion. With dispersion function being about $1 \mathrm{~m}$ and the momentum deviation about $1 \%$, the resulting amplitude change is $10 \mathrm{~mm}$, which is comparable to beam pipe radius. As a result, the momentum aperture is mainly limited by physical aperture.

The transverse emittance coupling factor has a direct impact on equilibrium beam dimensions, so the Touschek lifetime is a function of the coupling factor. As plotted in Fig. 24, Touschek lifetime does not change much with the coupling factor varied from 0.02 to 0.4 , although it reaches maximum when the coupling factor is about 0.125 . The average Touschek lifetime is $0.304 \mathrm{~h}, 0.310 \mathrm{~h}$, and $0.319 \mathrm{~h}$, respectively, for the three cases calculated.

\section{CONCLUSION}

The lattice design of TTX-II is presented. Two triplets of quadrupoles are placed in the straight sections between the baseline quadrupoles and bending magnets to help adjust ring parameters such as working point and dispersion. The horizontal dynamic aperture is over 10 times larger than the horizontal beam size at IP, for momentum spread between $-2 \%$ and $2 \%$. Frequency map analysis shows that for most of the particles within acceptance, the diffusion rate is low and long term stability can be expected. Beam lifetime is estimated to be $0.3 \mathrm{hr}$.

The beam evolution from injection to equilibrium is simulated. After injection, the beam undergoes dilution both transversely and longitudinally due to phase space mismatching. It takes a few tens of thousands turns for the beam to reach a quasiequilibrium state, after which, intrabeam scattering, synchrotron radiation, residual gas scattering, and Thomson scattering dominate the beam motion. The equilibrium horizontal emittance is estimated to be a few $\mu \mathrm{m}$ depending on the emittance coupling factor.

In most storage rings, chromaticity is corrected to be positive to avoid head-tail instability with mode number 0 . In our case, a conventional sextupole correction scheme is not viable. However, both particle tracking and analytical calculation indicate that the instability can be easily suppressed by Landau damping.

\section{ACKNOWLEDGMENTS}

This work is supported by Science Challenge Project No. TZ2018005.

[1] C. Tang, W. Huang, R. Li, Y. Du, L. Yan, J. Shi, Q. Du, P. $\mathrm{Yu}, \mathrm{H}$. Chen, T. Du et al., Tsinghua Thomson scattering X-ray source, Nucl. Instrum. Methods Phys. Res., Sect. A 608, S70 (2009).

[2] H. Xu, W. Huang, C. Tang, and S. Lee, Design of a 4.8-m ring for inverse Compton scattering x-ray source, Phys. Rev. ST Accel. Beams 17, 070101 (2014).

[3] W. Hardt, J. Jäger, and D. Möhl, Technical Report No. CMP00065010, 1982.

[4] F. Zimmermann, Tune shift with amplitude induced by quadrupole fringe fields, Technical Report No. CERN-SL2000-009-AP, 2000.

[5] M. Borland, in Proceedings of the 6th International Computational Accelerator Physics Conference, ICAP2000, 2000, Darmstadt, Germany (Argonne National Laboratory, IL, USA, 2000).

[6] H. Grote and F. Schmidt, in Proceedings of the 2003 Particle Accelerator Conference, Portland, OR (IEEE, New York, 2003), pp. 3497-3499.

[7] K. Hwang and S. Lee, Dipole fringe field thin map for compact synchrotrons, Phys. Rev. ST Accel. Beams 18, 122401 (2015).

[8] M. Studio, CST-Computer Simulation Technology, Bad Nuheimer Str 19, 64289 (2008).

[9] K. Flöttmann et al., Astra: A space charge tracking algorithm Manual, Version 3 (2011):2014.

[10] F. Sacherer, CERN Divisional Report No. CERN/SI-BR/ 72-5, 1972.

[11] A. W. Chao, Physics of Collective Beam Instabilities in High Energy Accelerators (Wiley, New York, 1993).

[12] M. Sands, The head-tail effect: an instability mechanism in storage rings, Technical Report SLAC-TN-69-008, 1969. 
[13] Y. Shobuda and K. Yokoya, Resistive wall impedance and tune shift for a chamber with a finite thickness, Phys. Rev. E 66, 056501 (2002).

[14] G. Rumolo and F. Zimmermann, Electron cloud simulations: beam instabilities and wakefields, Phys. Rev. ST Accel. Beams 5, 121002 (2002).

[15] A. W. Chao, K. H. Mess, M. Tigner, and F. Zimmermann, Handbook of Accelerator Physics and Engineering (World Scientific, Singapore, 1999).
[16] K. Ng, The equivalence of inverse Compton scattering and the undulator concept, Technical Report No. FERMILABFN-0840-AD, 2009.

[17] J. D. Bjorken and S. K. Mtingwa, Part. Accel. 13, 115 (1982)

[18] S.-Y. Lee, Accelerator Physics (World Scientific Publishing Company, Singapore, 2011).

[19] A. Piwinski, The Touschek effect in strong focusing storage rings, arXiv:physics/9903034. 